\title{
Experiences of formation control of multi-robot systems with the Null-Space-based Behavioral Control
}

\author{
Gianluca Antonelli
}

\author{
Filippo Arrichiello
}

\author{
Stefano Chiaverini
}

\begin{abstract}
In this paper, two experimental case studies performed with a multi-robot system made of 6 Khepera II mobile robots are presented. The experiments, performed at the laboratory LAI (Laboratorio di Automazione Industriale) of the Università degli Studi di Cassino, are aimed at testing the performances and the robustness of a behavior-based technique, namely the Null-Space-based Behavioral control (NSB), while executing different kinds of missions. In particular, the NSB approach, based on an inverse kinematic technique inherited by industrial manipulator applications, has been developed to control a generic team of autonomous vehicles and it has been implemented on a centralized architecture to control, at a kinematic level, a platoon of autonomous mobile robots.
\end{abstract}

\section{INTRODUCTION}

In the recent years, a growing interest in multi-robot systems has resulted in a massive development of theories and technologies concerning their control. The interest in this field is well justified by the several advantages that such systems present respect to single autonomous robots and it is supported by the improvements in technologies that make it possible the interaction and the integration among multiple systems. One of the main motivations for Multi-Robot Systems (MRSs) employing is that MRSs can increase the effectiveness of the system, that is, respect to a single autonomous vehicle or to a team of non cooperating robots, a MRS can better perform a mission in terms of time and quality, can execute tasks not executable by a single robot (i.e., moving a large object) or can take advantages of distributed sensing and actuation. Moreover, instead of building and using a single powerful robot, a multi-robot solution can be easier and cheaper, can provide flexibility to tasks execution and can make the system tolerant to possible robots' faults.

The first works on coordination among multiple agents were motivated by the study of biological systems and by application in computer graphics, i.e., in 1986 Reynolds [24] made a computer model for coordinating animal motion as bird flocks or fish schools. This pioneer work inspired significant efforts in the study of group behaviors [22], [17] and then in the study of multi-robot formation [30]. In the latest years, a great number of applications of MRS, differing in the typologies of vehicles and missions, have been proposed, i.e.,

Authors are listed in alphabetical order.

G. Antonelli, F. Arrichiello and S. Chiaverini are with the Dipartimento di Automazione, Elettromagnetismo, Ingegneria dell'Informazione e Matematica Industriale, Università degli Studi di Cassino, Via G. Di Biasio 43, 03043, Cassino (FR), Italy, \{antonelli,f.arrichiello,chiaverini\}@unicas.it

S. Chakraborti is with the Indian Institute of Technology, 721302, Kharagpur, India, u3mf1004@iitian.iitkgp.ernet.in multi robot systems made up of Underwater Autonomous Vehicles (AUVs) [15], [27], aerial vehicles [28], [25], and fleet of marine crafts [16], or applications like exploration and mapping [13], box pushing and transportation [29] or entertainment [20].

Some of the most common techniques used to achieve coordinated control of MSRs make use of biological inspiration; behavior based approaches, widely studied for mobile robotic applications [10], are useful to guide a multi-robot system in an unknown or dynamically chancing environment. These approaches give the system the autonomy to navigate in complex environments avoiding off-line path planning, using sensors to obtain instantaneous information of the environment and increasing the flexibility of the system. Among the behavioral approaches, seminal works are reported in the papers [12] and [9], while, lately, behavioral approaches have been applied to the formation control of multi-robot systems as in, e.g. [23], [18] and [11].

Among the multiple approaches proposed in literature, a behavior based approach to control one single mobile robot has been presented in [3], [2], namely the Null-Space-based Behavioral (NSB) control. The NSB differs from the other existing methods in the behavioral coordination method, i.e., in the way the outputs of the single elementary behaviors are assembled to compose the final behavior. In particular, the NSB uses a geometric, hierarchy-based composition of the tasks' outputs to obtain the motion reference commands to the robot that allow the system to exhibit robustness with respect to eventually conflicting tasks. In [6], [7], the NSB were extended to the control of multi-robot systems and in [4], [5] preliminary experimental results were reported. In this paper, emphasizing new experimental aspects, other missions with a platoon of up to 6 Khepera II mobile robots are presented and discussed.

The presented experiments are accompanied by the relative videos that can be found at the webpage:

http://webuser.unicas.it/lai/robotica/video/

\section{NUlL-SPACE-BASED BEHAVIORAL CONTROL}

Generally, a mission involving several robots may requires the accomplishment of several tasks at the same time. A common approach is to decompose the overall mission of the system in elementary tasks (or behaviors), solve them as they are working alone and, finally, combine the outputs of the single tasks to obtain the motion command to each robot. As discussed in [3], [2] the Null-Space-based Behavioral control differs from the other existing methods in the behavioral coordination method, i.e., in the way the outputs of the single 


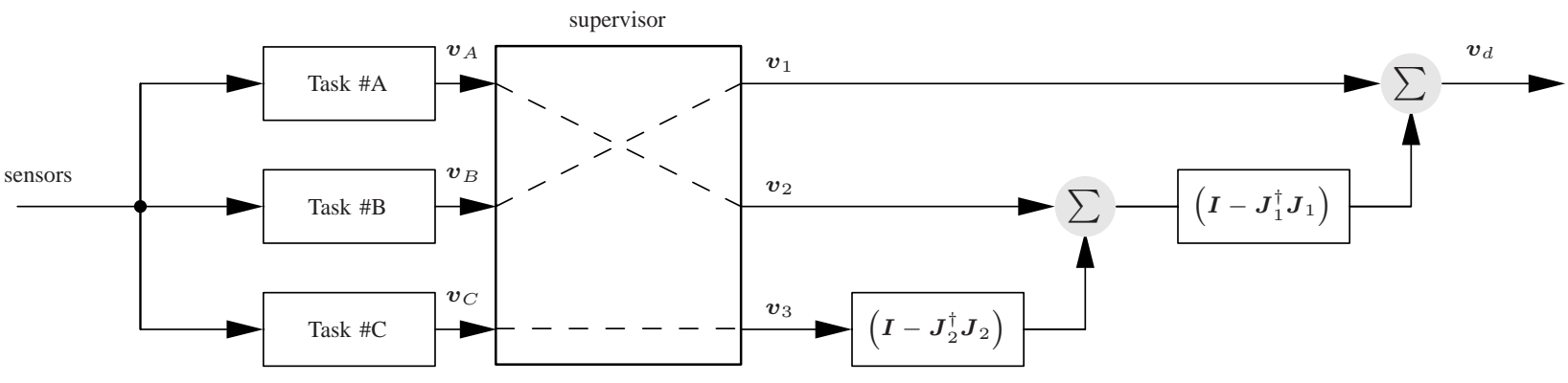

Fig. 1. Sketch of the Null-Space-Based behavioral control in a 3-task example. The supervisor is in charge of changing the relative priority among the tasks.

elementary behaviors are assembled to compose the final behavior. In particular, the NSB uses a geometric, hierarchybased composition of the tasks' outputs to obtain the motion reference commands for the robot that allow the system to exhibit robustness with respect to eventually conflicting tasks. The basic concepts are recalled in the following.

By defining as $\sigma \in \mathbb{R}^{m}$ the task variable to be controlled and as $\boldsymbol{p} \in \mathbb{R}^{l}$ the system configuration, it is:

$$
\boldsymbol{\sigma}=\boldsymbol{f}(\boldsymbol{p})
$$

with the corresponding differential relationship:

$$
\dot{\boldsymbol{\sigma}}=\frac{\partial \boldsymbol{f}(\boldsymbol{p})}{\partial \boldsymbol{p}} \boldsymbol{v}=\boldsymbol{J}(\boldsymbol{p}) \boldsymbol{v},
$$

where $\boldsymbol{J} \in \mathbb{R}^{m \times l}$ is the configuration-dependent task Jacobian matrix and $\boldsymbol{v} \in \mathbb{R}^{l}$ is the system velocity. Notice that $l$ depends on the specific robotic system considered, in case of a differential mobile robot $l=3$, and the term system configuration simply refers to the robot position/orientation, for a multi-robot system $l=3 n$ where $n$ is the number of robots, in case of a full actuated underwater vehicle $l=6$, finally, an anthropomorphic robots can reach very large value of $l$.

An effective way to generate motion references $\boldsymbol{p}_{d}(t)$ for the vehicles starting from desired values $\sigma_{d}(t)$ of the task function is to act at the differential level by inverting the (locally linear) mapping (2); in fact, this problem has been widely studied in robotics (see, e.g., [26] for a tutorial). A typical requirement is to pursue minimum-norm velocity, leading to the least-squares solution:

$$
\boldsymbol{v}_{d}=\boldsymbol{J}^{\dagger} \dot{\boldsymbol{\sigma}}_{d}=\boldsymbol{J}^{\mathrm{T}}\left(\boldsymbol{J} \boldsymbol{J}^{\mathrm{T}}\right)^{-1} \dot{\boldsymbol{\sigma}}_{d} .
$$

At this point, the vehicle motion controller needs a reference position trajectory besides the velocity reference; this can be obtained by time integration of $\boldsymbol{v}_{d}$. However, discretetime integration of the vehicle's reference velocity would result in a numerical drift of the reconstructed vehicle's position; the drift can be counteracted by a so-called Closed Loop Inverse Kinematics (CLIK) version of the algorithm, namely,

$$
\boldsymbol{v}_{d}=\boldsymbol{J}^{\dagger}\left(\dot{\boldsymbol{\sigma}}_{d}+\boldsymbol{\Lambda} \tilde{\boldsymbol{\sigma}}\right)
$$

where $\boldsymbol{\Lambda}$ is a suitable constant positive-definite matrix of gains and $\tilde{\sigma}$ is the task error defined as $\tilde{\sigma}=\sigma_{d}-\sigma$.
The Null-Space-based Behavioral control intrinsically requires a differentiable analytic expression of the tasks defined, so that it is possible to compute the required Jacobians. In detail, on the analogy of eq. (4), the single task velocity is computed as

$$
\boldsymbol{v}_{i}=\boldsymbol{J}_{i}^{\dagger}\left(\dot{\boldsymbol{\sigma}}_{i, d}+\boldsymbol{\Lambda}_{i} \tilde{\boldsymbol{\sigma}}_{i}\right),
$$

where the subscript $i$ denotes $i$-th task quantities. If the subscript $i$ also denotes the degree of priority of the task with, e.g., Task 1 being the highest-priority one, in the same case of 3 tasks we have provided as an example for the other two approaches considered, according to [14] the CLIK solution (4) is modified into

$$
\boldsymbol{v}_{d}=\boldsymbol{v}_{1}+\left(\boldsymbol{I}-\boldsymbol{J}_{1}^{\dagger} \boldsymbol{J}_{1}\right)\left[\boldsymbol{v}_{2}+\left(\boldsymbol{I}-\boldsymbol{J}_{2}^{\dagger} \boldsymbol{J}_{2}\right) \boldsymbol{v}_{3}\right]
$$

where $\boldsymbol{I}$ is the identity matrix of proper dimensions. Remarkably, eq. (6) has a nice geometrical interpretation. Each task velocity is computed as if it were acting alone; then, before adding its contribution to the overall vehicle velocity, a lower-priority task is projected onto the null space of the immediately higher-priority task so as to remove those velocity components that would conflict with it. Thus, the Null-Spacebased Behavioral control always fulfils the highest-priority task at nonsingular configurations. The lower-priority tasks, on the other hand, are fulfilled only in a subspace where they do not conflict with the ones having higher priority.

\section{TASK FUNCTION DEFINITIONS}

In this section, for lack of space, just a brief recalling of the definitions of task functions used to perform the subsequent experiments is presented. More details on the single task functions can be found in [8], [5].

\section{A. Centroid}

The centroid of a platoon expresses the mean value of the vehicles positions. In a 2 -dimensional case the task function is expressed by:

$$
\boldsymbol{\sigma}_{c}=\boldsymbol{f}_{c}\left(p_{1}, \ldots, p_{n}\right)=\frac{1}{n} \sum_{i=1}^{n} \boldsymbol{p}_{i} .
$$

where $\boldsymbol{p}_{i}=\left[\begin{array}{ll}x_{i} & y_{i}\end{array}\right]^{\mathrm{T}}$ is the position of the vehicle $i$. 


\section{B. Rigid formation}

The rigid formation task moves the vehicles to a predefined formation relative to the centroid. The task function is defined as:

$$
\boldsymbol{\sigma}_{f}=\left[\begin{array}{c}
\boldsymbol{p}_{1}-\boldsymbol{p}_{c} \\
\vdots \\
\boldsymbol{p}_{n}-\boldsymbol{p}_{c}
\end{array}\right],
$$

where $\boldsymbol{p}_{i}$ are the coordinates of the vehicle $i$ and $\boldsymbol{p}_{c}=\boldsymbol{\sigma}_{c}$ are the coordinates of the centroid.

\section{Obstacle avoidance}

With reference to a generic robot of the team, in presence of an obstacle in the advancing direction, the task function has to elaborate a driving velocity, aligned to the vehicleobstacle direction, that keeps the vehicle at a safe distance $d$ from the obstacle. Therefore, it is:

$$
\sigma_{o}=\left\|\boldsymbol{p}-\boldsymbol{p}_{o}\right\|
$$

where $\boldsymbol{p}$ and $\boldsymbol{p}_{o}$ are respectively the robot and the obstacle positions.

\section{EXPERIMENTAL SETUP}

The NSB were tested on a platoon of up to 7 Khepera II mobile robots available at the LAI (Laboratorio di Automazione Industriale) of the Università degli Studi di Cassino. The Khepera II, manufactured by K-team [1], are differentialdrive mobile robots with a unicycle-like kinematics and with an approximative dimension of $8 \mathrm{~cm}$ of diameter. Each Khepera has a Bluetooth turret that permits communication with the other robots or with external Bluetooth devices. In the proposed set-up, each robot communicates with a remote Linux-based PC, where a Bluetooth Dongle, building Virtual Serial Ports, allows the communication with up to 7 robots (see fig. 2). The remote Linux-based PC is aimed at implementing the NSB approach referring to a centralized structure.

Since the performed experiments focus on formation control, the vehicles positions are measured resorting to a vision-based system, made up of two high resolution color cameras and two frame-grabbers Matrox Metor II, running on a Windows-based PC. In particular, the upper turrets of each robot have a set of colored LEDs that are used to detect positions, orientations and identification numbers of each robot. The position measurements are performed at a sampling time of $100 \mathrm{~ms}$ while the estimation error has an upper bound of $\approx 0.5 \mathrm{~cm}$ and $\approx 1 \mathrm{deg}$. Moreover, the vision system permits to identify static obstacle (i.e., linear obstacles in fig. 2) or dynamic obstacles (i.e., a tennis ball) eventually present in the environment. The measurement are sent over the LAN to the Linux-based PC using the UDP/IP protocol.

Following the approach described in the previous Section, the NSB elaborates the desired linear velocity for each robot of the team. Being the Khepera unicycle-like robots, an heading controller has been derived from the controller reported in [19] to obtain wheels' desired velocities. Thus,

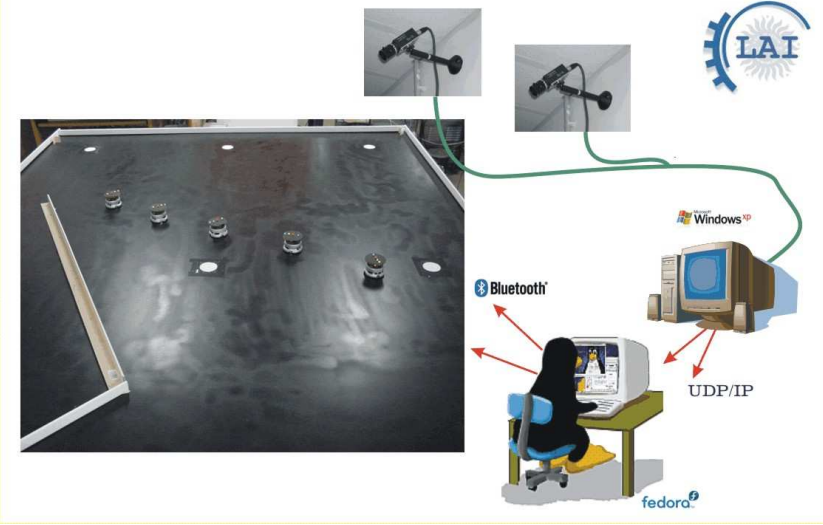

Fig. 2. Experimental set-up available at the LAI (Laboratorio di Automazione Industriale) of the Università di Cassino.

the remote Linux-based PC sends to each vehicle (through the Bluetooth module) the wheels' desired velocities with a sampling time of $120 \mathrm{~ms}$. The wheels' controller (on board of each robot) is a PID developed by the manufacturer. A saturation of $40 \mathrm{~cm} / \mathrm{s}$ and $180 \mathrm{deg} / \mathrm{s}$ has been introduced for the linear and angular velocities, respectively. Moreover, the encoders resolution is such that a quantization of $\approx 0.8 \mathrm{~cm} / \mathrm{s}$ and $\approx 9 \mathrm{deg} / \mathrm{s}$ are experienced.

\section{EXPERIMENTAL CASES STUDIES}

In this section, two experiments of formation control performed with the described experimental set-up are presented. In the first experiment, a team of robots have to reach and keep a linear formation avoiding a dynamic obstacle moving in the environment in an unpredictable way (i.e., a tennis ball pushed by hand). In the second experiments, the robots have to reach different circular formations while the relative positions of the robots dynamically switch and while a dynamic obstacle is moving through the circle.

In both the experiments, the mission is decomposed in three elementary task: avoid dynamic obstacles and collisions among the robots, keep the position of the centroid of the platoon at a constant value and keep a certain formation respect to the centroid (linear or circular).

\section{A. linear formation avoiding a dynamic obstacle}

In the first experiment, a platoon of 6 Khepera robots has to keep a linear formation (see fig.4) avoiding collisions among the robots and with a dynamic obstacles (a tennis ball pushed by hand) moving in the environment. In particular, the desired position of the centroid of the platoon is $\sigma_{c, d}=$ $\left[\begin{array}{ll}75 & 90\end{array}\right]^{\mathrm{T}} \mathrm{cm}$, the linear formation is rotated of 65 degrees respect to the axes $x$ and the robots have to keep a distance of $30 \mathrm{~cm}$ one from the others.

The priority of the 3 tasks implemented is:

1) obstacle avoidance

2) centroid

3) rigid linear formation. 

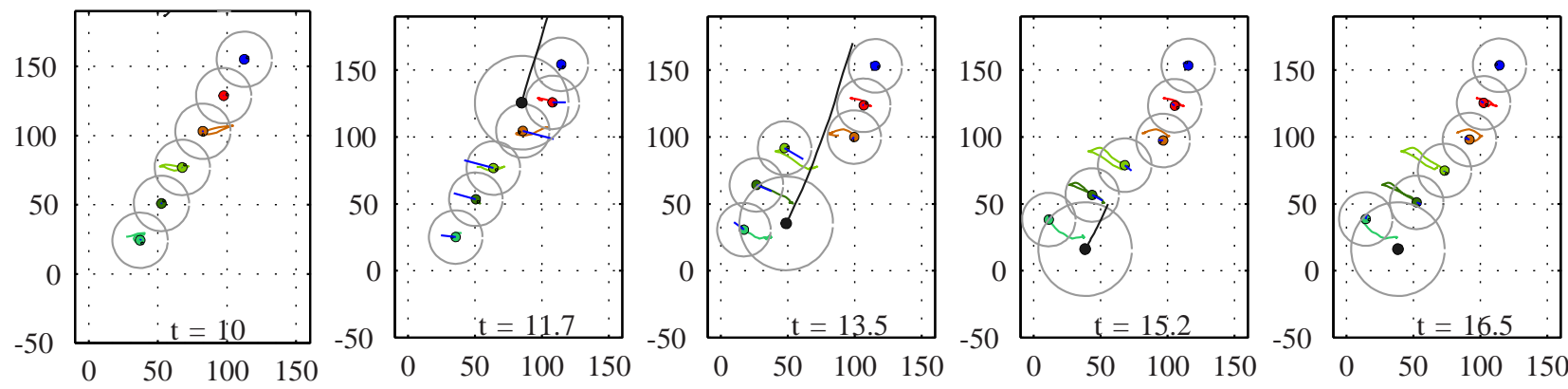

Fig. 3. Several steps of the linear formation mission while a tennis ball is passing through the formation.

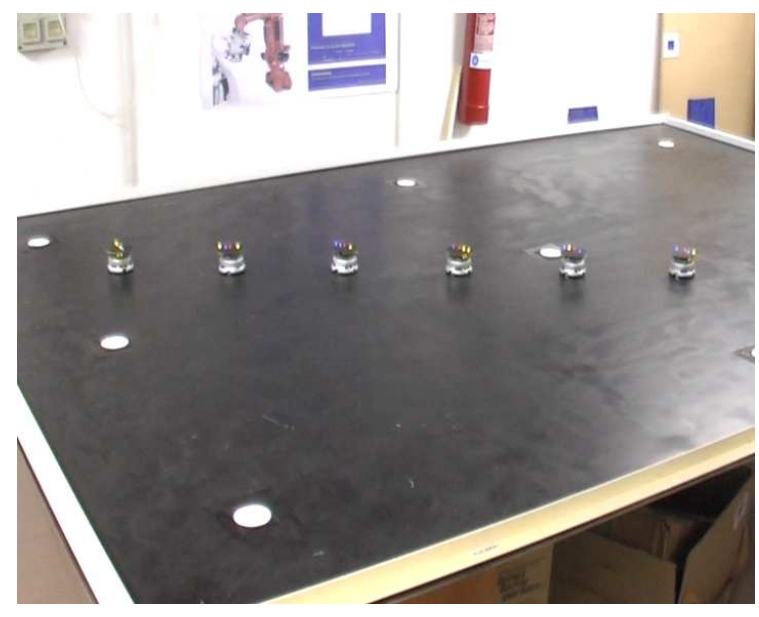

Fig. 4. Desired formation of the linear formation mission.

The gain matrix of the centroid task function is

$$
\boldsymbol{\Lambda}_{c}=\boldsymbol{I}_{2}
$$

while the gain matrix for the rigid formation task function $\Lambda_{f}$ is

$$
\boldsymbol{\Lambda}_{f}=2 * \boldsymbol{I}_{12}
$$

and the gain $\lambda_{o}$ of the obstacle avoidance task function is

$$
\lambda_{o}=1 \text {. }
$$

Moreover, the safety distance among the robots is $20 \mathrm{~cm}$ and the safety distance from the obstacle is $35 \mathrm{~cm}$.

Figure 3 shows several steps of the mission execution including the paths of the robots, the safety areas of the obstacle avoidance task function and the path of the dynamic obstacle. The robots start from the desired formation and keep it until an obstacle enter their safety area. When the dynamic obstacle is going through the formation, the robot have to avoid the obstacle to preserve their integrity, thus they temporary leave the desired formation and the centroid position. Once the obstacle has overtaken the formation, the robots do reach again the desired configuration avoiding collision among themselves. It is worth noticing that, in the last step of the mission, one of the robot is still close to the obstacle and does stay out of the formation, however the centroid is at the desired values. This behavior is due to the priority order of the tasks. In fact, at first the robot have to avoid collisions, then, in the null space of the obstacle avoidance task, they have to keep the centroid at the desired value, and finally, as a tertiary task, they have to reach the formation respect to the centroid. The conflict resolution policy applied by the NSB permits to guarantee the achievement of the lower-priority tasks only if they do not conflict with the higher ones, thus, in the specific configuration, the three task are conflicting and the last one (keeping the formation) can not be achieved. However, moving the ball far from the last robot, the three tasks do not conflict anymore and the formation can be reached again.

In fig. 3 only few significant steps of the mission are presented, however the relative video shows the whole mission.

\section{B. circular switching formation}

In the second experiments, a team of 6 robots have to dynamically reach circular formations while the relative positions of the robots switch, that is, once reached the desired configuration, each robot of the team has to exchange its position with the symmetrical one respect to the center of the circle (see fig.5). Recalling a situation similar to that one proposed by [21] for air traffic control, this mission permits to test the NSB while managing high traffic condition.

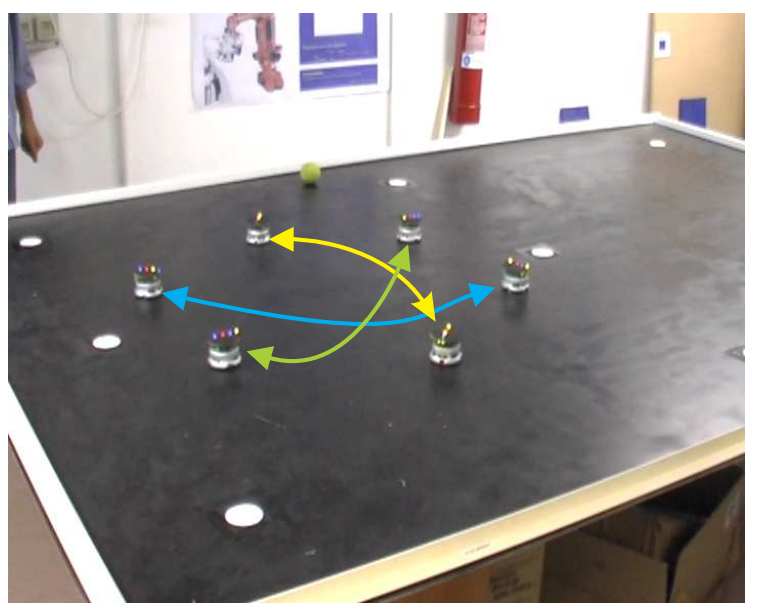

Fig. 5. Circular switching formation. 

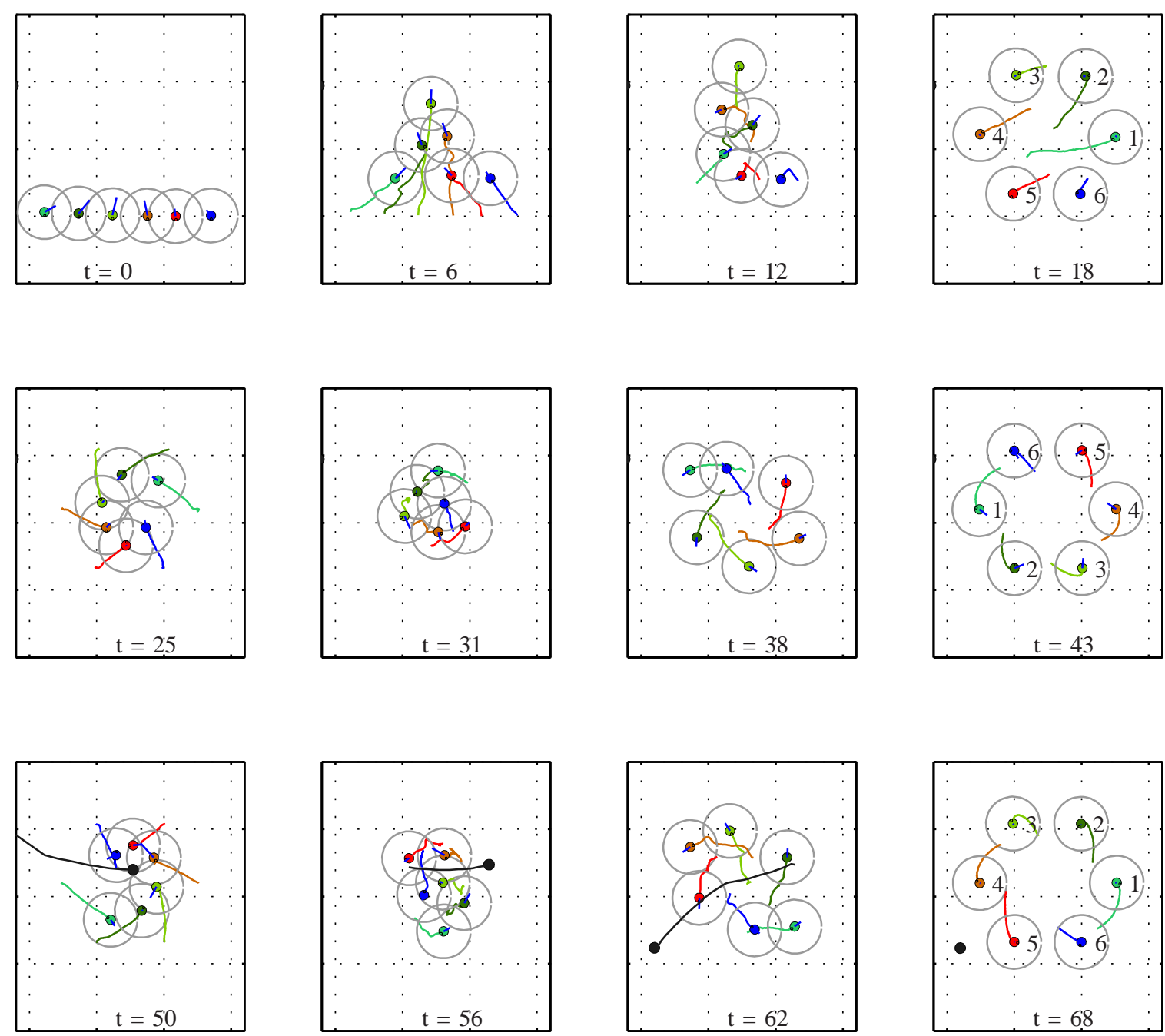

Fig. 6. Multiple steps of of the circular switching formation mission while a tennis balls is passing through the circle.

Also in this case, the priority of the 3 tasks implemented is:

1) obstacle avoidance

2) centroid

3) rigid circular formation

while, the gain matrix of the task functions are all Identity matrixes.

Figure 6 shows several steps of the performed mission. Starting from a linear configuration, the robots reach the circular formation in less the $20 \mathrm{~s}$. Then, once the error of the rigid formation task function has gone under a threshold value, the robots have to change their relative configuration. In particular, each robot has to exchange its position with its symmetrical respect to the center of the circle (as can be noticed observing colors and numbers of the robots in the steps 4,8 and 12 of fig. 6). During the change of formation, all the robots converges through the center of the circle increasing the risk of collisions and of the incurring of singular configurations. To significantly stress the algorithm, as shown by the last four steps of fig. 6, a tennis ball pass through the circle while the robot are changing the formation.
Also in this case the change is correctly achieved avoiding collision among the robots and with the obstacle. Thus, the correct achievement of the mission allows to consider the approach robust to high traffic condition and to conflict resolution.

Figures 7 and 8 show respectively the errors of the centroid task function and of the rigid formation task function. It is worth noticing that the centroid error is small during the all mission. However, it is not null because of the nonholonomy of the robots and of the eventual obstacle. In an ideal case of omnidirectional robots and ignoring the collision avoidance, the robot should keep the centroid in a constant position performing all the motions for changing the configuration in null space of the centroid task. Figure 7 shows that the change of formations are given as step functions and are correctly achieved also in presence of obstacles. In particular, it is possible noticing that the third change of formation takes longer and is more irregular than the previous ones because of the ball is passing through the circle. 


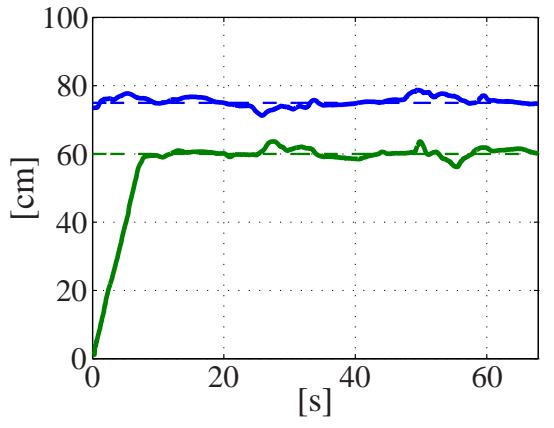

Fig. 7. Error of the centroid task function during the circular switching formation experiment.

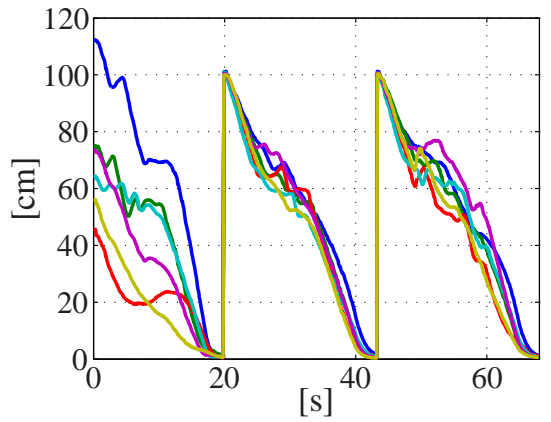

Fig. 8. Error of the rigid formation task function during the circular switching formation experiment.

\section{CONCLUSION}

Experimental results concerning the implementation of the Null-Space-based Behavioral approach to control a platoon of mobile robots were presented. The NSB approach allows to properly handle the outputs of several, eventual conflicting, behaviors/tasks. The experiments were performed at the LAI (Laboratorio di Automazione Industriale) of the Università degli Studi di Cassino equipped with 7 Khepera II mobile robots, 6 of which were used for the proposed experiments. The algorithm resulted in a successful implementation for dozen of missions requiring the movement in a quite cluttered environment.

\section{REFERENCES}

[1] http://www.k-team.com/. K-Team.

[2] G. Antonelli, F. Arrichiello, and S. Chiaverini. Experimental kinematic comparison of behavioral approaches for mobile robots. In Proceedings 16th IFAC World Congress, Prague, CZ, July 2005.

[3] G. Antonelli, F. Arrichiello, and S. Chiaverini. The Null-Space-based Behavioral control for mobile robots. In Proceedings 2005 IEEE International Symposium on Computational Intelligence in Robotics and Automation, pages 15-20, Espoo, Finland, June 2005.

[4] G. Antonelli, F. Arrichiello, and S. Chiaverini. Experiments of formation control with collisions avoidance using the Null-Space-based Behavioral control. In Proceedings 14th Mediterranean Conference on Control and Automation, Ancona, I, June 2006.

[5] G. Antonelli, F. Arrichiello, S. Chiaverini, and K. J. Rao. Preliminary experiments of formation control using the Null-Space-based Behavioral control. In Proceedings 8th IFAC Symposium on Robot Control, Bologna, I, Sept. 2006.
[6] G. Antonelli and S. Chiaverini. Kinematic control of a platoon of autonomous vehicles. In Proceedings 2003 IEEE International Conference on Robotics and Automation, pages 1464-1469, Taipei, TW, Sept. 2003.

[7] G. Antonelli and S. Chiaverini. Fault tolerant kinematic control of platoons of autonomous vehicles. In Proceedings 2004 IEEE International Conference on Robotics and Automation, pages 33133318, New Orleans, LA, April 2004.

[8] G. Antonelli and S. Chiaverini. Kinematic control of platoons of autonomous vehicles. IEEE Transactions on Robotics, 22(6):12851292, Dec. 2006.

[9] R.C. Arkin. Motor schema based mobile robot navigation. The International Journal of Robotics Research, 8(4):92-112, 1989.

[10] R.C. Arkin. Behavior-Based Robotics. The MIT Press, Cambridge, MA, 1998.

[11] T. Balch and R.C. Arkin. Behavior-based formation control for multirobot teams. IEEE Transactions on Robotics and Automation, 14(6):926-939, 1998.

[12] R.A. Brooks. A robust layered control system for a mobile robot. IEEE Journal of Robotics and Automation, 2:14-23, 1986.

[13] W. Burgard, M. Moors, C. Stachniss, and F.E. Schneider. Coordinated multi-robot exploration. IEEE Journal of Robotics, 21(3):376-386, June 2005.

[14] S. Chiaverini. Singularity-robust task-priority redundancy resolution for real-time kinematic control of robot manipulators. IEEE Transactions on Robotics and Automation, 13(3):398-410, 1997.

[15] E. Fiorelli, N.E. Leonard, P. Bhatta, D. Paley, R. Bachmayer, and D.M. Fratantoni. Multi-auv control and adaptive sampling in monterey bay. In Proceedings IEEE Autonomous Underwater Vehicles 2004: Workshop on Multiple AUV Operations, pages 134-147, Sebasco, ME, June 2004.

[16] I.A.F. Ihle, R. Skjetne, and T.I. Fossen. Nonlinear formation control of marine craft with experimental results. In Proceedings 43rd IEEE Conference on Decision and Control, volume 1, pages 680-685, Paradise Island, The Bahamas, Dec 2004.

[17] M.J. Mataric. Designing emergent behaviors: From local interaction to collective intelligence. In Proceedings of the International Conference on Simulation of Adaptive Behavior: From Animal to Animal, pages 432-441, 1992.

18] M.J. Mataric. Behavior-based control: Examples from navigation, learning, and group behavior. Journal of Experimental and Theoretical Artificial Intelligence, 9(2-3):323-336, 1997.

19] G. Oriolo, A. De Luca, and M. Vendittelli. WMR control via dynamic feedback linearization: design, implementation, and experimental validation. IEEE Transactions on Control Systems Technology, 10(6):835852, 2002.

[20] E. Pagello, A. D'Angelo, and E. Menegatti. Cooperation issue and distributed sensing for multirobot systems. Proceedings of the IEEE, 94(7):1370-1383, July 2006.

[21] L. Pallottino, E.M. Feron, and A. Bicchi. Conflict Resolution Problems for Air Traffic Management Systems Solved With Mixed Integer Programming. IEEE Transaction on Intelligent Transportation Systems, 3(1):3, 2002.

[22] L.E. Parker. Designing control laws for cooperative agent teams. In Proceedings 1993 IEEE International Conference on Robotics and Automation, volume 3, pages 582-587, Atlanta, GA, May 1993.

[23] L.E. Parker. On the design of behavior-based multi-robot teams. Advanced Robotics, 10(6):547-578, 1996.

[24] C. Reynolds. Flocks, herd and schools: A distributed behavioral model. Computer Graphics, 21(4):25-34, 1987.

[25] B. Seanor, Y. Gu, M.R. Napolitano, G. Campa, S. Gururajan, and L. Rowe. 3-aircraft formation flight experiments. In 14th Mediterranean Conference on Control and Automation, Ancona,I, July 2006.

[26] B. Siciliano. Kinematic control of redundant robot manipulators: A tutorial. Journal of Intelligent Robotic Systems, 3:201-212, 1990.

[27] D.J. Stilwell and B.E. Bishop. Platoons of underwater vehicles. IEEE Control Systems Magazine, 20(6):45-52, 2000.

[28] D.M. Stipanovic, G. Inalhan, R. Teo, and C.J. Tomlin. Decentralized overlapping control of a formation of unmanned aerial vehicles. Automatica, 40:1285-1296, 2004.

[29] H.G. Tanner, S.G. Loizou, and K.J. Kyriakopoulos. Nonholonomic navigation and control of cooperating mobile manipulators. IEEE Transactions on Robotics and Automation, 19(1):53-64, 2003.

[30] P.K.C. Wang. Navigation strategies for multiple autonomous robots moving in formation. Journal of Robotic Systems, 8(2):177-195, 1991. 